\title{
Tarsal Tunnel Syndrome Secondary to the Posterior Tibial Nerve Schwannoma
}

\author{
Jung Won Song ${ }^{1}$, Sung Han $\mathrm{Oh}^{1}$, Pyung Goo Cho', Eun Mee ${ }^{1}{ }^{2}{ }^{2}$ \\ Departments of ${ }^{I}$ Neurosurgey, ${ }^{2}$ Pathology, Bundang Jesaeng General Hospital, Seongnam, Korea
}

A 77-year-old female presented with complaint of burning pain and paresthesia along the medial aspect of ankle, heel and sole of the left foot. An ankle MRI, electromyelogram (EMG) with nerve conduction velocity (NCV) and pathologic findings were all compatible with Tarsal tunnel syndrome caused by the posterior tibial nerve Schwannoma. Operative release of the Tarsal tunnel and surgical excision of Schwannoma were performed under the microscopy. It is necessary to have a possible lump in mind when Tarsal tunnel syndrome is suspected, such as posterior tibial nerve Schwannoma.

Key Words: Posterior Tibial Nerve $\cdot$ Schwannoma $\cdot$ Tarsal Tunnel Syndrome

\section{INTRODUCTION}

Although Schwannomas are the most common peripheral nerve sheath tumor, Schwannoma of the posterior tibial nerve and it branch is a rare etiology causing Tarsal tunnel syndrome. We report a case of Tarsal tunnel syndrome caused by the posterior tibial nerve Schwannoma and mention surgical strategy with literature review.

\section{CASE REPORT}

A 77-year-old female presented with complaint of burning pain and paresthesia along the medial aspect of ankle, heel and sole of the left foot. The pain radiated to the toes. The patient mentioned that the pain felt like chestnut bur piercing from the medial aspect of the ankle to the arch of left foot. The symptom was aggravated for four years. The patient did not relate to any ankle trauma historical. Physical examination of the patient revealed a small palpable mass below the medial malleus of the left foot. A Tinel sign was typical on percussion of the posterior tibial nerve that sent radiating pain into the arch and toes of the left foot. However, she did not show weakness, nor atrophy of the intrinsic foot muscles. There were three neurofibromas on her trunk, however other evidence to

Corresponding author: Sung Han Oh, MD

Department of Neurosurgey, Bundang Jesaeng General Hospital, 20,

Seohyeon-ro 180 beon-gil, Bundang-gu, Seongnam-si, Gyeonggi-do 13590, Korea

Tel: +82-31-779-0280, Fax: +82-31-779-0895

E-mail: shoh1@dmc.or.kr diagnose neurofibromatosis was insufficient. An ankle magnetic resonance imaging (MRI) revealed about a $22 \times 19 \times 9 \mathrm{~mm}-$ sized ovoid soft tissue mass in the posterior ankle connected to the posterior tibial nerve. The mass lies beneath the flexor retinaculum of ankle and showed relatively strong enhancement (Fig. 1).

The NCV study showed no response sensory nerve action potentials of the left medial and lateral plantar nerves. Motor conduction study of the deep peroneal and tibial nerves was normal. However, needle EMG study was not showed abnormal spontaneous activities of left lower extremity including abductor digiti minimi and abductor hallucis muscles. The above electrophysiological diagnosis was compatible with Tarsal tunnel syndrome. Operative release of the Tarsal tunnel and surgical excision of the mass after incision of the neural sheath with the normal nerve bundles preserved were performed under the microscopy. The nerve was free of entrapment (Fig. 2). Pathologic finding revealed Schwannoma (Fig. 3). After the operation, her burning pain and paresthesia of the left ankle were somewhat relieved without any neurologic deficit.
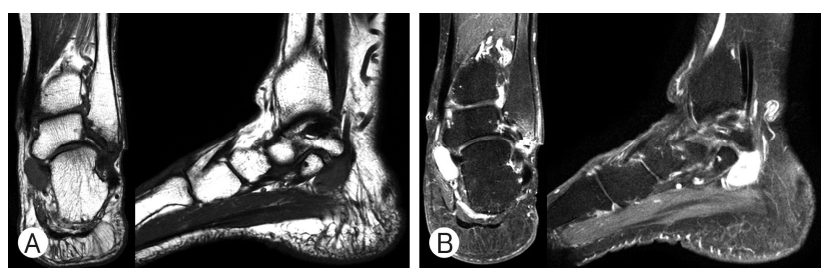

Fig. 1. Coronal and sagittal magnetic resonance images of the left ankle, which shows iso-signal intensity on the T1-weighted (A) and strong enhancement on the Gadolinium enhanced images (B). Note its relation to the posterior tibial nerve and the ovoid soft tissue mass with well formed capsule. 

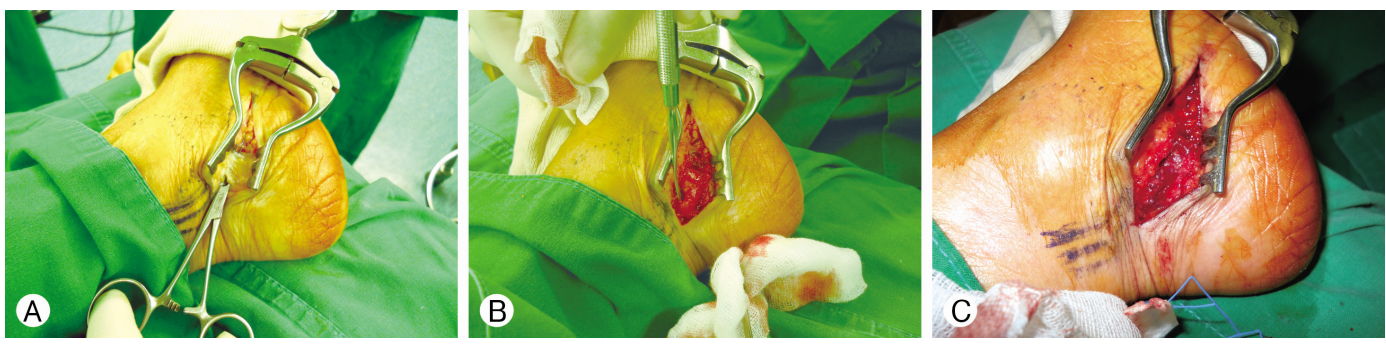

Fig. 2. Intraoperative photographs. After skin incision, the flexor retinaculum over the tarsal tunnel was identified and incised $(A)$, There was an ovoid mass of the posterior tibial nerve and the normal nerve proximal and distal to the tumor was fully exposed (B), and Incision of the neural sheath, the mass was completely removed and the neural sheath was repaired (C).
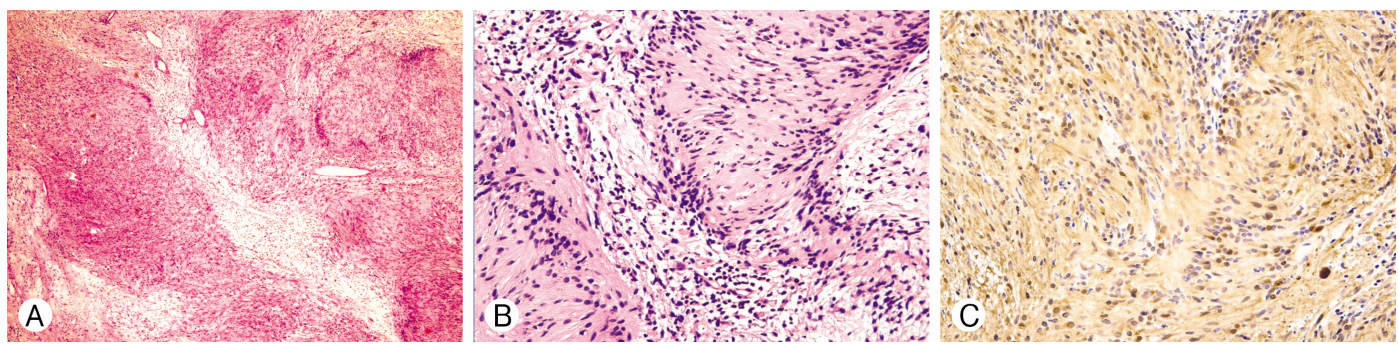

Fig. 3. Pathologic findings. The tumor shows alternating Antoni $A$ area (short fascicles with nuclear palisading) and Antoni B area (loosely textured area) (Hematoxylin and Eosin, $\times 40)(A)$, The tumor are composed of spindle cells with twisted nuclei and indistinct cytoplasmic borders (Hematoxylin and Eosin, $\times 200)(B)$, and The tumor cells show immunoreactivity for S-100 protein (S-100 protein. $\times 200)(C)$.

\section{DISCUSSION}

Tarsal tunnel syndrome is an entrapment neuropathy that flexor retinaculum compresses the posterior tibial nerve and its branch posterior to the medial malleus defined by Keck and $\mathrm{Lam}^{4}$. A positive Tinel sign is a key clue to suspect Tarsal tunnel syndrome. The EMG and other electrophysiological test are specific to diagnose the Tarsal tunnel syndrome and the NCV should be included because of its low sensitivity of $\mathrm{EMG}^{1,5)}$. There are various etiologies of Tarsal tunnel syndrome classified as internal and external factors ${ }^{2}$. The outcome of early diagnosis and treatment has been reported to be superior $^{2)}$. In this patient, her symptom had been aggravated for four years before the management, which is considered not to fully relieve her symptom. Thus, it has been suggested that in patients with some pain and abnormal sensation in the sole, more aggressive tests and management for Tarsal tunnel syndrome should be required ${ }^{7}$. On the other hand, Schwannoma is a benign, encapsulated, soft tissue tumor that arises from a peripheral nerve sheath ${ }^{1)}$. Although it can occur anywhere in the body, the foot is affected in only $10 \%$ of cases ${ }^{3)}$. Moreover, Schwannoma of the posterior tibial nerve producing secondary tarsal tunnel syndrome has rarely been reported ${ }^{3,5)}$. There are two possible reasons for the delay in that diagnosis ${ }^{6}$. First, a deep-seated swelling may escape detection by palpation in the thigh or the calf. Secondly, neuro- pathic pain expressed in the foot in the absence of a palpable lump may mislead the clinician, so that radiculopathy or entrapment neuropathy may be suspected ${ }^{6}$. Because a lump may not be palpable in the early phases, they recommended an examination of the entire length of the posterior tibial nerve, including the sciatic nerve by palpation and percussion? ${ }^{6}$. In this patient, a painful palpable lump in the ankle with a positive Tinel sign were the important clues to suspect some mass lesion, such as peripheral nerve tumor, causing the Tarsal tunnel syndrome. However, it took four years to diagnose it. In the posterior tibial nerve Schwannoma causing Tarsal tunnel syndrome, the treatment of choice is surgical excision and decompression of the nerve and its branches ${ }^{3)}$. In this patient, the flexor retinaculum was incised and the normal nerve proximal and distal to the tumor was adequately exposed. After incision of the neural sheath, a surgical plane between the tumor capsule and nerve bundles was removed whole and the tumor was totally excised under the microscope. There was a small fascicle entering the proximal pole of the tumor and was removed (Fig. 2). 


\section{CONCLUSION}

In conclusion, Schwannomas found in the lower extremity can also cause distal symptoms or injury, so they should always be considered as a differential diagnosis when tarsal tunnel syndrome is suspected. A painful palpable mass in the ankle with a positive Tinel sign are clues to suspect Tarsal tunnel syndrome. The EMG with NCV and imaging studies are essential not to misdiagnose possible Schwannoma causing the Tarsal tunnel syndrome. Early detection and surgical tactic mentioned in discussion are essential to improve the symptom without neurologic deficit.

\section{REFERENCES}

1. David WS, Doyle JJ: Segmental near nerve sensory conduction studies of the medical and lateral plantar nerve. Electromyogr Clin Neurophysiol 36:411-417, 1996

2. Hong JT, Lee SW, Son BC, Sung JH, Kim IS, Kim MC: Surgical Experiences of the Tarsal Tunnel Syndrome. J Korean Neurosurg Soc 36:443-447, 2004

3. Katrina H, Jessica V, Sarah D, Beverly FJ, John G: Tarsal Tunnel Syndrome Secondary to Schwannoma of the Posterior Tibial Nerve. The Journal of Foot \& Ankle Surgery 53:79-82, 2014

4. Lam SJ: The surgery of some strictures and stenoses. The tarsal tunnel syndrome. Ann R Coll Surg Engl 50:325-327, 1972

5. Mabin D: Distal nerve compression of the leg. Clinical and electrophysiologic study. Neurophysiol Clin 27:9-24, 1997

6. Nawabi DH, Sinisi M: Schwannoma of the posterior tibial nerve The Problem of Delay in Diagnosis. The Journal of Bone and Joint Surgery (Br) 89(B):814-816, 2007

7. Sammarco GJ, Chang L: Outcome of surgical treatment of tarsal tunnel syndrome. Foot Ankle Int 24:125-131, 2003 